\title{
Apreciação da Usabilidade do Moodle: Um Estudo de Caso no Contexto do Ensino Fundamental
}

\author{
Jéssica Tamires da Costa Penna ${ }^{1}$, Erica Rodrigues Oliveira ${ }^{2}$, Glívia Angélica \\ Rodrigues Barbosa ${ }^{3}$ \\ ${ }^{1}$ Instituto de Ciências Exatas e Informática - Pontifícia Universidade Católica de Minas \\ Gerais (PUC-MG). Av. Afonso Vaz de Melo, 1.200 - Barreiro de Baixo - Belo \\ Horizonte - MG - Brasil, CEP 30640-070 \\ ${ }^{2}$ Centro Universitário UNA. Av. Cristiano Machado, 11.157 - Belo Horizonte - MG - \\ Brasil, CEP 31744-007 \\ ${ }^{3}$ Departamento de Computação - Centro Federal de Educação Tecnológica de Minas \\ Gerais (DECOM/CEFET-MG). Av. Amazonas, 7675 - Nova Gameleira - Belo \\ Horizonte - MG - Brasil. \\ penna_jessica@hotmail.com, erica.oliveira@prof.una.br, \\ gliviabarbosa@decom. cefetmg.br
}

\begin{abstract}
Virtual environments provide learning support to students and other users a range of resources and tools to be used. The usability is one of the characteristics that should be considered when dealing with educational tools. Motivated by this context, the goal of this study was to assess the usability of Moodle from the perspective of elementary school students. The results pointed to usability issues that may prejudice the use of this platform for this profile users.
\end{abstract}

Resumo. Os ambientes virtuais de apoio ao aprendizado proporcionam aos alunos e demais usuários uma gama de recursos e ferramentas a serem utilizados. A usabilidade do sistema é uma das características que deve ser considerada quando tratamos de ferramentas educacionais. Motivados por esse contexto, o objetivo deste trabalho consistiu em apreciar a usabilidade do Moodle na perspectiva de alunos que cursam o ensino fundamental. Os resultados apontaram para problemas de usabilidade que podem dificultar o uso dessa plataforma por esse perfil de usuários.

\section{Introdução}

A inserção de software como ferramenta para apoiar a aprendizagem dos alunos é uma realidade em várias instituições de ensino. Neste sentido, o Moodle, que é um Ambiente Virtual de Aprendizagem (AVA), que possui recursos para compartilhamento de materiais, realização de testes e avaliações e aprendizado colaborativo (e.g., fóruns, chats), se popularizou [Abed 2010] devido às suas características nativas e por ser um software livre de código aberto, que permite customizações [Moodle, 2015]. Segundo os dados divulgados pela própria plataforma, o Brasil é o terceiro maior utilizador do Moodle no mundo [Moodle, 2015]. 
Apesar do crescente uso do Moodle, especialmente no Brasil, não foram encontrados na literatura trabalhos sobre a utilização dessa ferramenta no ensino fundamental brasileiro, bem como apreciação da usabilidade de sua interface por este público. Com o objetivo de contribuir para a pesquisa nesta área, este trabalho realizou uma avaliação da usabilidade da plataforma Moodle, como uma ferramenta complementar à aprendizagem em aulas presenciais de alunos, crianças e adolescentes, do ensino fundamental (i.e., alunos de $1^{\circ}$ ao $9^{\circ}$ ano). Para isso foi realizado um estudo de caso, em que o Moodle foi avaliado sob as perspectivas de especialistas em Interação Humano Computador (IHC) e de crianças e adolescentes do ensino fundamental em um colégio de Belo Horizonte.

Esse tipo de avalição é importante porque dentre os desafios envolvidos no projeto e avaliação de software para o domínio educacional, destacam-se a necessidade de avaliar a aprendizagem dos alunos, além da qualidade da interação, e a necessidade de oferecer apoio aos alunos durante a realização de atividades [de Almeida e Azevedo 2009].

Nesse sentido, os resultados desta pesquisa apontaram para problemas de usabilidade tanto nos recursos nativos do Moodle, quanto nas customizações, que podem dificultar, ou até mesmo inviabilizar, o uso do Moodle, por crianças e adolescentes, como uma ferramenta de complemento à aprendizagem em aulas presenciais do ensino fundamental.

Em termos de contribuições esse trabalho é relevante para duas áreas distintas: informática na educação e interação humano-computador (IHC). Para a área de informática na educação contribui à medida que fornece indicadores que devem ser considerados durante a construção de um sistema de apoio ao aprendizado, de forma a melhorar a usabilidade e, consequentemente, a satisfação, eficiência e produtividade dos usuários. Para a área de IHC contribui com orientação aos projetistas do Moodle a melhorar sua interface.

$\mathrm{Na}$ próxima seção são apresentados os trabalhos relacionados. Na sequencia o estudo de caso realizado é descrito, tanto em relação à metodologia adotada, bem como em relação aos principais resultados. Por fim, as conclusões e os próximos passos deste trabalho são discutidos.

\section{Trabalhos Relacionados}

Nesta seção apresentamos alguns trabalhos existentes que tratam da avaliação do Moodle, com o objetivo de melhorar sua interface e contribuir com a aprendizagem dos alunos. Os trabalhos apresentados avaliam o Moodle sob diferentes aspectos, como comunicabilidade [Capelão et al., 2001], acessibilidade [Jatobá et al., 2009; Rezende, 2007], usabilidade [Magalhães e Gomes, 2010] e uso no meio corporativo [Nunes e Torres, 2012].

Com o objetivo de avaliar a comunicabilidade do Moodle, Capelão et al. (2011) aplicaram o Método de Inspeção Semiótica (MIS) [Barbosa e Silva, 2010] e o Método de Avaliação de Comunicabilidade (MAC) [Barbosa e Silva, 2010]. A avaliação foi feita com usuários surdos e ouvintes, no Moodle da Universidade Federal de Minas Gerais. Como resultado os autores observaram que o Moodle apresenta problemas de 
comunicação que comprometeram o uso de algumas funcionalidades, tanto por usuários surdos, quanto por ouvintes.

Alguns trabalhos focaram na avaliação da acessibilidade do Moodle por pessoas com necessidades especiais [Jatobá et al., 2009; Rezende, 2007]. Em ambos os trabalhos observou-se que o Moodle pode ser utilizado como um facilitador da inclusão digital para esse tipo de usuário, entretanto ainda é necessário fazer algumas adaptações em sua interface para pessoas com necessidades especiais.

Magalhães et al. (2010) avaliaram a usabilidade no Moodle IFAM (Instituto Federal de Educação Ciência e Tecnologia - Amazonas). Para esta avaliação, foram utilizados os métodos do percurso cognitivo e o MAC [Barbosa e Silva, 2010]. Observou-se que parte dos problemas identificados podem ser resolvidos conforme as habilidades dos administradores do Moodle. Com isso, os autores sugerem a necessidade de definir boas práticas de usabilidade na configuração do Moodle.

Nunes e Torres (2012) realizaram uma pesquisa que analisou os recursos oferecidos pelo Moodle, enquanto ferramenta para apoio a aprendizagem organizacional, para capacitar recursos humanos que atuam dentro da Universidade Federal de Santa Catarina (UFSC). Constatou-se que a aprendizagem organizacional pode ser apoiada nos recursos do Moodle, desde que sejam observados alguns aspectos, como por exemplo, incentivo ao uso de ferramentas como chats e grupos de discussão.

O presente trabalho se difere dos demais uma vez que, ao invés de focar na aplicabilidade do Moodle para apoiar no ensino de jovens e adultos, ele foca na avaliação da usabilidade da plataforma Moodle sob a perspectiva de crianças e adolescentes que cursam o ensino fundamental (i.e., alunos do $1^{\circ}$ ao $9^{\circ}$ ano). A partir dessa abordagem foi possível apreciar a usabilidade do Moodle como uma ferramenta complementar à aprendizagem em aulas presenciais do ensino fundamental considerando esse perfil de usuários.

\section{Estudo de Caso - Metodologia e Principais Resultados}

Para atingir o objetivo proposto foi realizado um estudo de caso em três etapas. A etapa inicial consistiu em inspecionar a interface do Moodle com o intuito de identificar os potenciais problemas de usabilidade que poderiam ser vivenciados por crianças e adolescentes do ensino fundamental, essa avaliação foi conduzida por especialistas em IHC. A segunda etapa consistiu em apreciar o uso dessa plataforma por alunos de um colégio do ensino fundamental em Belo Horizonte, Minas Gerais. Finalmente a última etapa consistiu em triangular os resultados obtidos entre as duas avaliações.

A triangulação refere-se a um procedimento da pesquisa qualitativa realizada através da comparação de dados extraídos por diferentes métodos, cujo intuito é conferir a validade dos resultados obtidos em uma pesquisa científica [Cho \& Trent, 2006]. Neste caso, a validação consiste em buscar diferentes interpretações para a mesma questão de pesquisa. Em outras palavras, seu objetivo não é replicar resultados, mas sim, verificar se os mesmos são coerentes, plausíveis e consistentes [Cho \& Trent, 2006]. Na próxima seção caracterizamos o ambiente onde o estudo de caso foi conduzido. 


\subsection{Ambiente do Estudo de Caso - Moodle do Colégio Master}

O Colégio Master é uma instituição privada de ensino fundamental com aproximadamente 400 alunos. Nessa instituição professores e alunos fazem uso da plataforma Moodle desde o ano de 2008. A plataforma é utilizada como uma ferramenta complementar para apoio ao aprendizado, uma vez que visa estender as práticas pedagógicas aplicadas em sala de aula.

Através do Moodle os alunos realizam atividades como: provas, trabalhos e avaliações multidisciplinares. Além disso, eles acessam o material didático e colaboram entre si, através de fóruns e chats. Os professores, por sua vez, tem acesso aos recursos, tanto para acompanhar seus alunos, quanto para colaborarem entre si.

Quando o Moodle foi implantado no Colégio, os professores receberam um treinamento oferecido pela empresa terceirizada que customizou o sistema para a instituição. No entanto, os alunos não recebem nenhum tipo de treinamento específico. Eles apenas obtêm um informativo contendo login e senha e o primeiro acesso é realizado juntamente com a coordenação pedagógica e equipe de Tecnologia da Informação (TI) do Colégio. Na próxima seção é apresentada a metodologia, bem como os resultados da primeira etapa do estudo de caso.

\subsection{Avaliação da Usabilidade sob a Perspectiva de Especialistas}

Uma das formas de analisar a usabilidade de um sistema é realizar a Avaliação Heurística (AH) que é um método consolidado para avaliar interfaces [Barbosa e Silva, 2010]. A AH é um método de inspeção, realizado por especialistas em IHC, para identificar potenciais problemas de usabilidade. Através desse método o especialista julga a conformidade da interface em relação a um conjunto de 10 heurísticas de usabilidade, propostas por Nielsen [1993]. Para cada problema identificado o avaliador associa uma ou mais heurísticas violadas e atribui um grau de gravidade. A escala de gravidade compreende valores entre 0 e 4, em que quanto maior o valor, mais grave o problema de usabilidade no sentido de inviabilizar o uso do sistema [Nielsen, 1993] [Barbosa e Silva, 2010].

Nessa etapa, seguiu-se os passos da $\mathrm{AH}$, com o objetivo de identificar os principais problemas de usabilidade que os alunos do ensino fundamental poderiam vivenciar. Para isso foram considerados os seguintes cenários, considerados frequentes e críticos para esses alunos: (1) acesso ao site por meio de login/logout, (2) acesso à disciplina, (3) download de arquivo, (4) fórum, (5) questionário que inclui exercícios e provas, (6) upload de arquivo/imagem e (7) utilização da ferramenta de edição de textos do Moodle. A avaliação foi realizada na primeira quinzena de setembro de 2014 por dois avaliadores com experiência na aplicação da $\mathrm{AH}$ e os resultados foram validados por um especialista na área de IHC (i.e., profissional da área com mais de cinco anos de experiência). A seguir os principais resultados dessa avaliação são apresentados e discutidos.

\subsubsection{Análise dos Potenciais Problemas Identificados}

Durante a avaliação, foi encontrado um total de dezesseis problemas que violaram pelo menos uma heurística. Por exemplo, na tarefa 1 (acesso ao site por meio de login/logout) identificou-se a ausência da usabilidade para o usuário efetuar login no 
Moodle. Quando o usuário digita o login e/ou senha errados não são exibidas mensagens de feedback. Isso impossibilita que o usuário identifique e corrija seu erro. Esse foi considerado um problema com gravidade 04 , uma vez que sem autenticação não é possível acessar o sistema.

Uma vez que cada problema poderia ser associado à ausência de uma ou mais heurísticas de usabilidade e que uma mesma heurística poderia ser violada em diferentes problemas, para realizar a análise proposta e apresentar os resultados de forma consolidada, verificou-se quantas heurísticas de usabilidade foram violadas e com que frequência (i.e., incidência) essas violações aconteciam. A tabela 01 apresenta os resultados consolidados.

Tabela 01 - Consolidação dos Problemas

\begin{tabular}{l|c|c}
\hline \multicolumn{1}{c|}{ Heurística de Usabilidade Violadas pelo Moodle } & \multicolumn{2}{c}{$\begin{array}{c}\text { Incidências (Quantos } \\
\text { problemas violaram) }\end{array}$} \\
\hline Visibilidade do estado do sistema & 4 & $25 \%$ \\
\hline Correspondência entre o sistema e o mundo real & 8 & $50 \%$ \\
\hline Controle e liberdade do usuário & 3 & $19 \%$ \\
\hline Consistência e padronização & 6 & $38 \%$ \\
\hline Prevenção de erro & 4 & $25 \%$ \\
\hline Reconhecimento e recuperação de erros & 4 & $25 \%$ \\
\hline Reconhecimento em vez de memorização & 8 & $50 \%$ \\
\hline Flexibilidade e eficiência de uso & 5 & $31 \%$ \\
\hline Design estético e minimalista & 5 & $31 \%$ \\
\hline Ajuda e documentação & 5 & $31 \%$ \\
\hline
\end{tabular}

Após a consolidação dos problemas, verificou-se que todas as heurísticas de usabilidade foram violadas pelo menos uma vez. Por meio da Tabela 01 verifica-se que as principais heurísticas violadas foram "Correspondência entre o sistema e o mundo real", "reconhecimento em vez de memorização" e "consistência e padronização".

A frequência similar de problemas violando essas três heurísticas pode estar associada à relação existente entre as mesmas. Isso porque, se o projetista não faz uso de elementos em sua interface que façam analogia ao mundo real, dificilmente ele consegue manter uma consistência na interface e o usuário tem dificuldade de reconhecer as possibilidades de interação através do sistema e, ao invés disso, ele memoriza como interagir. Esses problemas comprometem outro princípio de usabilidade, referente ao "controle e liberdade do usuário", uma vez que, se o usuário não conhece as possibilidades de interação ele fica limitado durante o uso do sistema.

As heurísticas de "prevenção de erros", "reconhecimento e recuperação de erros" e "ajuda e documentação" também foram violadas. Nota-se, então, que no ambiente estudado não é recorrente o uso de recursos que visam evitar e ajudar o usuário a solucionar seu erro.

Finalmente, verificou-se que o Moodle, muitas vezes, não apresenta feedback e o design de suas telas contêm informações desnecessárias, que não são frequentemente 
utilizadas e faz o usuário ficar perdido e sem saber qual ação tomar. Essas informações irrelevantes deveriam ser trocadas pelo uso de aceleradores ou atalhos, para permitir a interação mais rápida e eficiente dos usuários. Esses problemas descritos são causados pela violação das heurísticas "visibilidade do estado do sistema", "flexibilidade e eficiência de uso" e "design estético e minimalista".

\subsection{Avaliação da Usabilidade do Moodle sob a Perspectiva da Opinião dos Usuários - Aplicação de Questionários}

Com o objetivo de coletar a opinião dos usuários sobre a usabilidade do Moodle do Colégio Master, foi aplicado um questionário online, composto por 18 questões de múltipla escolha. As questões foram agrupadas de forma que fosse possível caracterizar: (1) o perfil do participante da pesquisa e a (2) opinião dos usuários sobre a usabilidade da ferramenta.

A aplicação do questionário ocorreu no período de seis dias, durante o mês de outubro de 2014. O mesmo foi divulgado e compartilhado publicamente em uma das disciplinas do ambiente estudado para acesso dos alunos. Ao todo 105 alunos responderam ao questionário.

Destes participantes, todos são alunos do colégio a partir do $5^{\circ}$ ano. Sendo que do $5^{\text {a }}$ ano $(20 \%)$, do $6^{\circ}$ ano $(28 \%)$, do $7^{\circ}$ ano $(19 \%)$, do $8^{\circ}$ ano $(20 \%)$ e do $9^{\circ}$ ano $(13 \%)$. Suas idades variam entre 10 e 15 anos. A divisão de gêneros dos participantes foi 53\% do sexo masculino e $47 \%$ do sexo feminino. Em relação ao tempo de uso do Moodle, uma quantidade significativa dos participantes (40\%) utiliza a ferramenta há pelo menos de 01 ano, enquanto que, $22 \%$ utiliza há mais de 02 anos, $16 \%$ já utiliza o Moodle há mais de 03 anos e $22 \%$ utiliza a ferramenta há mais de 04 anos.

Em relação à usabilidade do Moodle os usuários foram convidados a responder questões sobre a utilização do AVA, relacionadas aos potenciais problemas identificados durante a Avaliação Heurística. Quando questionados sobre a usabilidade dos recursos relacionados à "prevenção de erros" e "recuperação de erros", pelo menos $60 \%$ dos alunos afirmaram que o sistema não apresenta nenhuma mensagem sobre o erro ou que evite que o mesmo ocorra. O usuário deve descobrir o erro sozinho, pois não há auxílio na identificação e recuperação do problema.

Durante a avaliação heurística observou-se que a padronização de telas do sistema possui informações irrelevantes que poderiam causar dúvidas de entendimento nos usuários. Nesse sentido, os usuários foram questionados se encontraram dificuldades durante a interação com o Moodle de forma que fosse necessário executar a mesma tarefa repetidas vezes. A pesquisa apontou que $61 \%$ dos participantes tiveram que realizar a mesma tarefa mais de uma vez para entender como era o funcionamento do Moodle, fato atribuído a falta de padronização na interface.

Os usuários também foram indagados se o sistema utiliza palavras e conceitos que são familiares, ou seja, se há correspondência entre o sistema e o mundo real. Porém, $70 \%$ dos participantes afirmaram que as expressões do sistema são de difícil entendimento. Por isso, esses usuários declararam que, ao invés de reconhecer os elementos da interface, é preciso memorizar e guardar as informações durante a interação. Tal observação representa um problema de usabilidade, uma vez que, quem deveria propiciar essa transição de forma transparente e orientar em que estado do 
sistema o usuário se encontra é o próprio AVA. Finalmente, como apontado na avaliação dos especialistas, mais da metade dos participantes da pesquisa (51\%) afirmou que não há uma documentação de ajuda no Moodle que os auxilie no uso da ferramenta.

Finalizada a análise do questionário, constata-se que, no contexto educacional, os problemas aqui confirmados podem dificultar o uso do Moodle pelos alunos do ensino fundamental. Isso porque, além de se preocupar com o aprendizado de um conteúdo, o aluno precisa também aprender e lidar com os problemas de usabilidade da ferramenta.

\subsection{Avaliação da Usabilidade do Moodle sob a Perspectiva da Experiência de Uso dos Usuários - Teste com Usuários}

Com o intuito de consolidar os resultados obtidos com a avaliação heurística e a aplicação de questionários, realizou-se um teste com usuários. Este teste foi realizado com usuários representativos do sistema, para apreciar a experiência de uso do Moodle. Neste teste, foram mensurados valores como o tempo gasto para a realização das tarefas propostas pelo teste, bem como as dificuldades encontradas e facilidades de uso.

Foram selecionados quatro alunos do ensino fundamental, com idade entre 09 a 15 anos. Segundo Nielsen (2000), no contexto de avaliações na área de IHC, recomenda-se que pelo menos três usuários participem da avaliação. Todos os participantes foram submetidos à realização de 07 tarefas com um avaliador acompanhando o teste. Essas tarefas são executadas frequentemente pelos alunos no Moodle e apresentaram potenciais problemas durante a Avaliação Heurística.

O teste aconteceu no laboratório de informática do Colégio no mês de outubro de 2014. Os computadores utilizados tinham o sistema operacional Windows XP e os usuários poderiam escolher entre os navegadores Internet Explorer, Mozilla ou Google Chrome para realizar o teste. $\mathrm{O}$ estado inicial do sistema era a área de trabalho com o ícone dos três navegadores à escolha do usuário.

O tempo por tarefa começava a ser contado a partir do momento em que o aluno recebia os documentos com instruções sobre as tarefas e parava de ser cronometrado quando o aluno dissesse que finalizou ou desistiu da tarefa. A conclusão das tarefas era marcada com três tipos de tags: (1) executou sem dificuldades, (2) executou com dificuldade e (3) executou parcialmente (i.e, não finalizou). A tabela 02 apresenta $o$ tempo médio de execução das tarefas.

Tabela 02 - Tempo médio de execução das tarefas

\begin{tabular}{|c|c|c|c|c|c|c|c|}
\hline TAREFA & 1 & 2 & 3 & 4 & 5 & 6 & 7 \\
\hline DESCRIÇÃO & Login & $\begin{array}{c}\text { Navegação } \\
\text { entre cursos }\end{array}$ & $\begin{array}{c}\text { Recurso } \\
\text { Livro }\end{array}$ & Fórum & $\begin{array}{c}\text { Recurso } \\
\text { calendário e } \\
\text { comentários }\end{array}$ & Questionário & Logout \\
\hline $\begin{array}{c}\text { MÉDIA DE } \\
\text { TEMPO } \\
\text { (em minutos) }\end{array}$ & $01: 25$ & $00: 27$ & $00: 35$ & $00: 36$ & $01: 15$ & $03: 30$ & $01: 16$ \\
\hline
\end{tabular}

Considerando o perfil de um usuário iniciante, o tempo estimado de conclusão do teste (i.e., todas as tarefas) foi de 15 minutos, nenhum usuário estourou o tempo total, uma vez que o tempo médio de execução foi de 08:04 minutos. Porém alguns usuários demandaram mais tempo, quando comparados entre si, para realização de 
tarefas simples. O usuário 04, por exemplo, na tarefa 01 (acesso ao site e login) levou o dobro da média de tempo dos demais usuários, pois não sabia qual site acessar e teve dificuldade de encontrar o botão de acesso ao sistema.

Em relação à execução das tarefas, observou-se que na tarefa 02 o usuário deveria entrar na disciplina criada para a avaliação chamada "Teste com o Usuário". Apesar de estar, aparentemente visível, na lista de exibição de disciplinas, apenas um aluno clicou na disciplina sem fazer uma busca detalhada.

A tarefa 03 consistiu em fazer o upload de um arquivo. Todos os usuários realizaram a tarefa sem perguntas ao avaliador e com a média de tempo semelhante. A tarefa 04 comtemplava o recurso de Livro do Moodle. Um usuário executou a tarefa parcialmente (i.e, não conseguiu finalizar), pois encontrou dificuldades durante a interação prevista para concluir a tarefa. Apesar dos outros usuários concluírem a tarefa, todos relataram que o caminho de interação e as possibilidades de navegação disponíveis geram dúvidas, por não existir mensagens de feedback que orientem na execução da tarefa.

A tarefa 05 estava relacionada com o recurso do Fórum. Todos conseguiram realizar a tarefa, porém alguns alunos tiveram mais dificuldades. As queixas sobre esse recurso estavam relacionadas ao excesso de informação apresentado. $\mathrm{O}$ usuário pode clicar em diferentes lugares para responder o questionário e o bloco descrito como "assunto" é desnecessário, uma vez que o fórum descreve um assunto específico. Além disso, um usuário relatou que não se sentiu seguro ao submeter suas respostas no fórum, pois o sistema não apresenta feedback se as réplicas foram salvas. $\mathrm{O}$ mesmo usuário comentou que falta um botão de retorno ao curso após o envio das respostas.

A tarefa 06 representava três funções do sistema: upload de imagens, uso da ferramenta de edição e envio de um questionário ou atividade no Moodle. Todos os participantes concluíram essa tarefa parcialmente. O sistema induz o usuário ao erro por não informar como ele deve fazer o upload de imagem. A maioria dos usuários copiava uma imagem da web e quando colava na barra de textos conseguia visualizá-la. No entanto, após encerrar o questionário e enviá-lo para a correção o sistema não valida essa imagem e mostra apenas um [X]. Foi possível observar que não existe prevenção de erros sobre esse fato e que falta uma documentação que, de fato, auxilie o usuário a efetuar o upload corretamente.

A tarefa 07 englobava os recursos de "Calendário", "Lista de Participantes", "Comentários" e a saída do sistema (logout). Dois usuários encontraram dificuldades ao utilizar o calendário e um terceiro, apesar de informar a resposta correta, disse que não concordava com a utilidade deste recurso. As outras funcionalidades não geraram dúvidas e todos concluíram facilmente.

Finalizados os testes, é possível concluir que, os problemas observados nas tarefas 2, 5 e 7, embora de menor gravidade, devem ser verificados. Já as barreiras de usabilidade detectadas nas tarefas que foram executadas parcialmente, 1, 3 e 6, devem ser consideradas como um alerta para melhoria na interface. Isso porque a falta de conhecimento em realizar o upload do arquivos/imagens, a dificuldade em editar textos através da ferramenta e a falta de documentação e mensagens de ajuda podem comprometer o uso da Moodle e, consequentemente, o aprendizado e a nota do aluno. 


\section{Conclusão e Trabalhos Futuros}

Nesse trabalho, a questão de pesquisa consistiu em apreciar a usabilidade do ambiente virtual de aprendizagem Moodle no Colégio Master, considerando o perfil de alunos do ensino fundamental (i.e., alunos do $1^{\circ}$ ao $9^{\circ}$ ano). A apreciação consistiu em avaliar essa propriedade sob a perspectiva de especialistas em IHC e contrastar os resultados obtidos com a avaliação realizada com alunos do colégio que utilizam essa ferramenta em seu cotidiano escolar.

A partir das análises realizadas e da triangulação entre as mesmas foi possível diagnosticar falhas de usabilidade e propor sugestões de melhorias para o bom funcionamento do sistema e para que o AVA apoie os alunos na aprendizagem.

Assim, algumas sugestões de melhorias são propostas. A primeira, diz respeito à disposição e quantidade de informação exibida ao usuário. Os recursos se apresentam sob uma forma de difícil visualização. Isso prejudica a usabilidade, e também a acessibilidade, devido a sua disposição em conjunto, em um espaço inadequado à quantidade de informação.

A segunda sugestão é para que haja uma padronização textual, pois as expressões e conceitos utilizados atualmente geram dúvidas nos alunos. É importante haver correspondência entre o sistema e o mundo real. Para isso, as expressões devem ser de fácil entendimento e compreensão para os alunos. Desta maneira, esses usuários poderão se concentrar unicamente na aprendizagem do conteúdo, ao invés também de ter que lidar constantemente com as dificuldades de uso do Moodle.

Outro ponto a ser discutido está relacionado à documentação e a ajuda on-line, necessária e, ao mesmo tempo, ausente no Moodle estudado. O fato do sistema apresentar muita informação desencoraja a interação do usuário. A proposta é apresentar a informação de ajuda de maneira mais sucinta e objetiva, se possível com links que redirecionem para mais informações e exemplos de uso.

Os resultados apresentados e discutidos nesse trabalho são importantes tanto para melhorar a interface do Moodle avaliado no estudo de caso, quanto para incentivar a realização de treinamentos mais aprofundados para que todos os recursos oferecidos de fato auxiliem no processo de aprendizado dos alunos do ensino fundamental.

Contudo, é importante destacar que a relevância do trabalho não se limita a essa contribuição, isso porque inicialmente, a metodologia adotada neste estudo de caso pode ser reproduzida na avaliação do Moodle em outros contextos ou ainda na avaliação de outros AVAs. De forma análoga, os problemas de usabilidade aqui identificados podem auxiliar os projetistas de interface na melhoria desse tipo de ferramenta, bem como chamar atenção dos mesmos para que evitem esses problemas em novas versões desenvolvidas. Conclui-se, então, que o trabalho apresenta contribuições metodológicas e práticas para as áreas de Educação e IHC.

Sendo assim para futuros trabalhos seria interessante implementar as melhorias na interface para potencializar a usabilidade e, posteriormente, fazer o cruzamento de dados e averiguar se as mudanças foram positivas. Além da implementação das melhorias citadas, visando detectar problemas diferentes, é recomendado que seja realizada avaliação com professores. Tal avaliação permitirá delimitar a ampliação ou 
reformulação do que está sendo oferecido aos alunos para tornar o Moodle um ambiente virtual de interação e interatividade, compartilhando o conhecimento entre os usuários.

\section{Referencias}

ABED (2010). Censo 2010. Relatório Analítico de Aprendizagem a Distância no Brasil. http://www.abed.org.br/censoead/CensoEaDbr0809_portugues.pdf. Acesso fev 2015.

Barbosa, S.D.J.; Silva, B.S. (2010). Interação Humano-Computador. Elsevier Editora.

Capelão, L.; Coutinho, F.; Pereira, K.; Prates, R. (2011). Avaliação de comunicabilidade do Moodle para usuários surdos e ouvintes. In: Proc. IHC of IHC + CLIHC 2011, SBC, (2011)

Cho, J. \& Trent, A. (2006). Validity in qualitative research revisited. Qualitative Research, 6(3):319--340.

de Almeida, P. H. B; Azevedo, R. (2009) Modelos mentais: um estudo de caso referente à introdução de computadores no ensino. In: WIE. XXIX CSBC. Bento Gonçalves, RS.

Franciscato, F. T.; Ribeiro, P. S.; Mozzaquatro, P. M.; Medina, R. D. (2008) Avaliação dos Ambientes Virtuais de Aprendizagem Moodle, TelEduc e Tidia - Ae: um estudo comparativo. Revista Novas Tecnologias na Educação. Vol. 6, nº 2.

Jatobá, A.; Vrabl, S.; Barros, D. R.; Engelbrecht, A.; Braganholo, V. (2009) Ampliando horizontes de aprendizagem: ensaios sobre o uso do Moodle por deficientes visuais. In: XIII Congreso Internacional de Educación a Distancia, Chile.

Magalhães, E. et al. (2010) Impacto da Usabilidade na Educação a Distância:Um Estudo de Caso no Moodle IFAM. Artigos Industriais. IHC 2010- IX Simpósio de Fatores Humanos em Sistemas Computacionais.Out.,2010,BeloHorizonte.MG,

Moodle (2015). Moodle - modular object-oriented dynamic learning environment. https://moodle.org/. Acesso em maio 2015.

Mozzaquatro, M. P. and Medina, D. R. (2008). Avaliação do ambiente virtual de aprendizagem moodle sob diferentes visões: aspectos a considerar. Revista Novas Tecnologias na Educação. Vol. 6, nº 2 .

Nielsen, J. (1993) Usability Engineering. San Diego: Academic Press.

Nielsen, J. (2000) Why You Only Need to Test with 5 Users. Disponível em http://www.nngroup.com/articles/why-you-only-need-to-test-with-5-users/ Acesso em maio/2015.

Nunes, C. S., Torres, M. K. L., et. al. (2012). O ambiente virtual de aprendizagem Moodle: recursos para os processos de Aprendizagem Organizacional. SBIE. RJ.

Rezende, A. L. A. (2007). "Easy: Mediando as interações dos deficientes visuais com o ambiente virtual de aprendizagem Moodle.”, In: CIEEE 07: Anais do VII Congresso Iberoamericano de Informática Educativa Especial. 